\title{
Präsentationen des Fremden in der tschechischen Musik zwischen Anziehung und Abstoßung. Eine Studie tschechischer Fremd- und Selbstbilder ${ }^{1}$
}

\author{
Lenka Ǩ̌UPKOVÁ \\ Department of Musicology, \\ Faculty of Arts of Palacký University Olomouc \\ Univerzitní 3, 77180 Olomouc, Czech Republic \\ E-mail: lenka.krupkova@upol.cz
}

(Received: May 2018; accepted: September 2018)

\begin{abstract}
The Czechs have limited personal experience with foreign non-European cultures, people of different appearances and other religions. Apart from the not always latent xenophobic attitude towards "other" cultures, Czechs are known to have an almost paranoid fear of the decisions of larger nations. These are two complementary factors that determine the cultural profile of the Czech nation. Czech history, rich in moments and periods of the nation's failure, its humiliation and frustration, provides numerous examples serving to explain this situation. Everything foreign, new and unknown attracts an audience and at the same time repels it. This study demonstrates, using several examples from Czech music, how ambivalent the perception of "the other" can be: as something that fascinates but at the same time evokes fear and a feeling of threat. This experience with the ambivalent meaning of "the other" is surely not only characteristic of Czechs. Other nations also view "the other" as a projection wall of their desires and fantasies as well as fears. One can find similar motifs in other art works of a different provenience. In the case of small nations, however, these themes can be accentuated by the influence of particular historical situations and viewed from the perspective of established interpretations.
\end{abstract}

Keywords: Czech music, foreign cultures, perception, self-reflection

Mit fremden außereuropäischen Kulturen, mit Menschen anderen Aussehens und anderen Glaubens haben die Tschechen nur relativ wenige eigene Erfahrungen. Tschechien gehört trotz offener innereuropäischer Grenzen, trotz zunehmender globaler Mobilität und Migration zu den national und ethnisch sehr homogenen

1. Erstellung und Herausgabe wurden ermöglicht mit finanzieller Unterstützung der Philosophischen Fakultät der Palacký-Universität Olmütz im Jahre 2015, mit dem Fonds zur Unterstützung wissenschaftlicher Tätigkeit. 
Ländern. Es hat nur wenige und sehr kleine Minderheiten, vor allem Roma, Slowaken, Ukrainer, Polen und Vietnamesen. In Tschechien mit seinen ca. 10,5 Millionen Einwohnern leben dauerhaft nur etwa eine halbe Million Ausländer. Eine mit den mittleren und größeren Städten vergleichbare Vielfalt an Herkunftsländern und Kulturen ihrer Menschen findet man in Tschechien nicht.

Neben einer immer häufiger offen xenophoben Haltung gibt es in Tschechien geradezu paranoid ausgeprägte Ängste dieser zahlenmäßig eher kleinen Nation vor den Entscheidungen größerer Nationen, denen sie sich dann unterzuordnen hätte. Dies sind zwei, das kulturelle Profil der tschechischen Nation mitprägende komplementäre Faktoren, die jeweils im Zusammenhang miteinander erklärt werden müssen. Der Schlüssel dazu liegt vor allem in der tschechischen Geschichte, die reich an Augenblicken und Epochen nationaler Misserfolge, Demütigungen und Frustrationen ist. Einer der bekanntesten dieser Augenblicke ist die Schlacht am Weißen Berg im Jahr 1620. In dieser Schlacht wurde ein Aufstand des tschechischen protestantischen Adels von habsburgisch geführten, katholischen Heeren besiegt. Als Folge kam es anschließend zur fast kompletten Auslöschung des alten tschechischen Adels, außerdem zur Rekatholisierung der Länder der böhmischen Krone. Langfristig, für die nächsten rund 300 Jahre, wurden Böhmen und Mähren von Österreich einverleibt und zur politischen Provinz degradiert. Der tschechische Schriftsteller Alois Jirásek nannte die 300 tschechischen Jahre unter Habsburg „temno“ - die Zeit der Finsternis. Diese Zeit wurde Quelle vieler weiterer, in der tschechischen Literatur auftauchenden Mythen. Sie handeln meist von mutigen Böhmen und Mährern, die von rücksichtslosen, meist deutschsprachigen Fremdlingen niedergewalzt werden. Der Fluch des „Weißen Berges“ wurde auch im 20. Jahrhundert immer wieder beschworen: 1938 gilt als Datum des „Münchner Verrats“, der die Zerschlagung der Tschechoslowakei durch Nazi-Deutschland vorbereitete. Das Jahr 1948 ist der Nation als Datum der putschartigen, aus Moskau gesteuerten kommunistischen Machtübernahme in Erinnerung. 1968 ist einerseits das Jahr des „Prager Frühlings“, des tschechoslowakischen Versuchs einer politischen Liberalisierung des sozialistischen Systems; gleichzeitig markiert es die anschließende Besetzung der Tschechoslowakei durch die sowjetischen und weiteren ausländischen, also fremden Armeen.

Aber nicht immer wurde die Begegnung mit der Welt jenseits der politischen und sprachlichen Grenzen als Bedrohung des tschechischen Volkes betrachtet. Der Kontakt mit fremden Kulturen half den Tschechen wie anderen Nationen auch, ihren Egozentrismus und Ethnozentrismus zu mäßigen und sich selbst einen Spiegel vorzuhalten. ${ }^{2}$ Faszination und Staunen über Fremdes und Neugier nach Exotischem finden sich bereits bei den ersten tschechischen Reisenden und ihren

2.Zdeněk Hrbata, „Území exotiky“ [Das Gebiet der Exotik], in Cizí, jiné, exotické v české kultuře 19. stoleti [Das Fremde, das Andere und das Exotische in der tschechischen Kultur des 19. Jahrhunderts], hrsg. von Kateřina Piorecká und Václav Petrbok (Praha: Academia, KLP, 2008), 9-10. 
Reiseberichten aus dem Ausland. Sie sind ein Zeugnis dafür, dass ihr mittelalterlicher Autor auf Reisen ,schaut“ (divá se) und sich wundert (diví se), also neugierig, offen und fasziniert ist. Aus dem gleichen Wortstamm für die Wörter "schauen" (divat se) und "sich wundern" (divit se) erwächst im Tschechischen jedoch noch ein drittes frequentiertes Wort dieser reisenden Autoren: divný (seltsam, komisch, rätselhaft). ${ }^{3}$ Dívá se a diví se divným: Ein aus entfernten Weltwinkeln angereister Mensch schaut und wundert sich über rätselhafte, also unbekannte und daher verdächtige Dinge.

Das Fremde, Neue und Unbekannte zieht seine Beobachter an und stößt sie gleichzeitig ab. In dieser Studie versuche ich daher einige Beispiele in der tschechischen Musik dafür zu finden, wie die Fremdwahrnehmung in ihrer Ambivalenz zum Thema wird: als Faszination, aber auch als Angst und Gefühl der Bedrohung durch das Fremde.

\section{1.}

Ähnlich wie in anderen europäischen Musikkulturen der Romantik bedienten sich auch die tschechischen Komponisten in ihren Werken der exotischen Fremdheit mit Vorliebe als Inspirationsquelle für Handlungsentwürfe und ihre musikalische Verarbeitung. Im ersten Teil meines Vortrags möchte ich Ihnen einige Werke tschechischer Komponisten vorstellen, in denen die Verwendung exotischer Motive von Relevanz ist.

In der Kunst des 19. Jahrhunderts geht es noch nicht so sehr um die Demonstration authentischer Exotismen. Die Pflicht, mit beinahe wissenschaftlicher Methodik die Kunst entfernter Völker oder die ursprüngliche Volkskultur des eigenen Volkes zu erforschen, empfanden Künstler erst mit Beginn des 20. Jahrhunderts. Der Musik der Epoche der Romantik geht es um „die Rezeption gewisser exotischer Momente" ${ }^{4}$ doch handelt es sich eher um Stilzitate, die dem zeitgenössischen Bild der Musikstruktur angepasst wurden und nicht weiter störten. Die Komponisten schöpfen aus dem Repertoire vorgebildeter Klischees und Stereotypen. ${ }^{5}$

Ein beliebter Exotismus des 19. Jahrhunderts ist das sogenannte Zigeunertum. Beispielsweise sind Auftritte sogenannter Zigeuner in der Oper, im Tanztheater oder im Schauspiel ein wirksames Mittel zur Vergrößerung der Vielfalt; musikalisch wird das häufig mit spanischem oder ungarischem Kolorit substituiert. Das stereotype Bild der so genannten Zigeuner ist das der Zirkusleute, der fahrenden

3. Ebda., 12.

4. Marta Ottlová-Milan Pospíšil, „K repertoáru exotismů v české hudbě 19. stoleti““ [Zum Repertoire des Exotismus in der tschechischen Musik des 19. Jahrhunderts], in Cizí, jiné, exotické, 339.

5. Ebda., 341. 
Tänzer, Jongleure und Tierdompteure, wie etwa die Figur Esmeralda Salamankas in Smetanas Prodaná nevěsta (Die verkaufte Braut). Ein oft reproduzierter Zigeunerinnentyp ist die Wahrsagerin; diese darf nur entweder jung und schön oder aber alt und hässlich aussehen - nicht zuletzt war dann auch das Stereotyp der gefährlich erotischen Zigeunerin besonders populär. Außerhalb von Opernwerken findet man den Zigeunerexotismus der tschechischen Musik auch im Rahmen der Vokalmusik. Ein Beispiel hierfür sind die dichterischen Zyklen Adolf Heyduks (1835-1923). Seine Cigánské melodie (Zigeunermelodien) und Nové cigánské melodie (Neue Zigeunermelodien) wurden von Karel Bendl, Antonín Dvořák und auch Vítězslav Novák vertont. Als musikalische Stereotype werden sogenannte Zigeunertonleitern mit den Intervallen der vergrößerten Sekunde und Quarte oder auch der Cimbal-Stilisierung verwendet. ${ }^{6}$

Im Liederzyklus Zápisník zmizelého (Tagebuch eines Verschollenen) von Leoš Janáček finden wir keines dieser musikalischen Klischees. Der Liederzyklus wurde 1917-1919 auf Grundlage eines anonymen Gedichts komponiert, das im Mai 1916 in den Lidové noviny (Volkszeitung) unter dem Titel „Aus der Feder eines Autodidakten" abgedruckt worden war. Dieses Werk gehört zwar zur Musik des 20. Jahrhunderts, allerdings korrespondiert die ursprüngliche Kompositionsstruktur, wie auch in anderen Werken dieses Komponisten, nicht sehr stark mit der Handlungsebene. Diese bleibt dem Geist der Romantik des 19. Jahrhunderts verhaftet. Janáčeks Zefka verkörpert das oben beschriebene romantische Stereotyp der Zigeunerin als gefährlich verführerischer, wilder und eigentlich unzivilisierter Frau. Sie stammt aus fremden, unbekannten Landstrichen und führt den tüchtigen tschechischen Burschen Janíček in ebensolche unbekannte Gegenden fort. Janíček verlässt die Konventionen, die Sicherheit und die Ordnung seiner heimatlichen Gemeinschaft für immer.

Der Zyklus besteht aus 22 Liedern, in denen der junge Mann Janek - Janíček (Tenor) sein Schicksal erzählt. In der Mitte des Zyklus, in den Liedern Nr. 9-11, tritt in diesen monologischen Fluss das dynamische Element der Geschichte ein: die Zigeunerin Zefka. Im 10. Lied kommt ein Chor dreier kommentierender Frauen hinzu. Der Vers des 11. Liedes Von der Heidin Wangen Zauberduft weht so... kommt es zum Bruch der bisherigen Handlung. Der bis dahin reine und naive Knabe wird von der attraktiven und lüsternen Zigeunerin Zefka verführt:

TENOR: Von der Heidin Wangen Zauberduft weht so zart.

ALT: „Willst mich schlafen sehen, ganz nach Zigeunerart?

Willst mich sehen? Ganz nach Zigeunerart?“ 
TENOR: Räumt sie ein Steinchen weg, einen Ast zur Seite:

„Schon ist das Bett gemacht! schon zur Nacht bereitet!“

\begin{abstract}
ALT: „Polster der Waldboden,
Decke des Himmels Zier, und kühlt der Tau die Hände, wärm ich im Schoß sie mir.“
\end{abstract}

TENOR: Trug ein Röckchen nur, lag auf dem Boden dort.

Ach wie da jammernd wich, ach wie da jammernd wich meine Keuschheit fort. ${ }^{7}$

Im Schlusslied wird der Held der Geschichte mit den Folgen seines Abenteuers konfrontiert. Für immer folgt er seiner Zigeunerin und ihrem gemeinsamen Sohn in die Fremde, weit weg von seiner Heimat.

Einen ähnlichen ungebundenen Zigeunerinnentyp repräsentiert Emilia Marty, die Titelheldin der Janáček-Oper Věc Makropulos (Die Sache Makropulos, Uraufführung: 1926). Das Libretto dieser Oper verfasste Janáček selbst, und zwar nach einem Theaterstück des tschechischen Schriftstellers Karel Čapek. Auf der Bühne entwickelt sich die Geschichte zu einem Krimi: es geht um den gerichtlichen Streit um das Erbe bzw. um den Diebstahl eines Testaments, das das Rezept eines Jugendelixiers verspricht. Emilie muss und will dieses Elixier dringend wieder zurückhaben. Die Geschichte mündet schließlich in die Klärung der Schicksalsfrage, ob es überhaupt gut ist, ewig zu leben. Mittendrin erscheint unversehens ein gewisser Hauk auf, ein verrückter Alter. Ihn hat vor 50 Jahren die Zigeuner-Sängerin Eugenia Montez, alias Emilia Marty verführt und um den Verstand gebracht. Selbst in seiner geistigen Umnachtung erkennt er die berühmte Operndiva:

HAUK: Die Zigeunerin liebt' ich dort die, man „chula negra“ nannte, nämlich weit unten in Andalusien.

Wer sie sah, verliebte sich, rief: Gitana, hoch!

Wer sie sah, verliebte sich, rief: Gitana, hoch!

Ich ließ ihr dann alles dort, wirklich alles!

Und blieb für's ganze Leben dann schon wie blöd, Sie verstehen doch? ${ }^{8}$

7. Deutsche Übersetzung von Max Brod.

8. Deutsche Übersetzung von Max Brod. 
„Chula negra“ ist spanisch und ist eine sexistisch-rassistisches Schimpfwort für Frauen mit dunkler Haut und dunklen Haaren. Die Zigeunerin, sowieso ein Konstrukt männlicher erotischer Fantasie, wird hier auch noch zur Projektionsfläche für männliche Hass- und Versagensgefühle.

Ein dankbares exotisches Motiv ist für tschechische Landratten seit jeher das Meer, besonders als aufgewühltes Naturelement. Dieses Sujet verarbeitete der tschechische Dichter Svatopluk Čech in der lyrischen Meeresfantasie Bouře (Der Sturm). Sie inspirierte 1910 den Komponisten Vítězslav Novák zu einer weitläufigen Komposition, einer mehr als einstündigen gleichnamigen Kantate. Um dem Werk ausreichende Authentizität zu verleihen, scheute der Komponist nicht davor, zur Inspiration an die Nordsee zu reisen..$^{9}$ In deren brausenden Wogen ertrank er dann beinahe. Das acht Kapitel lange Gedicht vertonte Novák zu einer Reihe von Solo-Auftritten sich abwechselnder Chöre. Diese sind miteinander verbunden durch weitläufige orchestrale Zwischenspiele, die lautmalerisch das Tosen des Meeres darstellen. In scharfem Kontrast zum sich steigernden Wogen der Wellen erklingt im fünften Teil des Gedichts, „In der Kajüte“, das Wiegenlied eines Schwarzafrikaners. Verbindungen des Meeres und der Figur des Schwarzen Mannes sind wiederum ein beliebtes exotisches Element. In den böhmischen Ländern bzw. in Österreich-Ungarn, also Ländern ohne ausgeprägte Kolonialgeschichte, hatten sie eine besonders starke Wirkung. Im Fall der Kantate Bouře bemühte sich der Komponist aber zunächst um größtmögliche Authentizität. Er begann daher ethnographische Liedersammlungen aus Madagaskar zu studieren. ${ }^{10} \mathrm{Da}$ er in ihnen kein zufriedenstellendes Material fand, konstruierte er den eigenen Worten nach eine eigene, quasi-exotische Tonleiter. Aus diesem musikalischen Konstrukt heraus entsteht ein exotistisches Motiv, das im orchestralen Fluss ständig wiederkehrt. Konkret handelt es sich um die Folge von acht Tönen, in der sich Ganz- und Halbton abwechseln. Novák fügte hier jedoch das Intervall einer vergrößerten Sekunde zwischen die dritte und vierte Stufe der Skala ein (c-d-es-fis-g-a-b-c), ansonsten hätte er sich einem oktatonischen Modus angenähert. ${ }^{11}$ Den exotischen Charakter und den Eindruck ,primitiver Musik"12 sollen die begleitenden leeren Quinten im Bass und die Instrumentierung eines sich ostinatisch wiederholenden Motivs im Englischen Horn hervorrufen.

9. Er schildert das Erlebnis ausführlich in seiner Autobiographie: Vítězslav Novák, O sobě a o jiných [Über sich und andere] (Praha: Supraphon, 1970), 157-159.

10. Vladimír Lébl, Vitězslav Novák, život a dílo [Vítězslav Novák, Leben und Werk] (Praha: ČSHV, 1964), 133.

11. Olivier Messiaen ordnete den oktatonischen Modus später als zweiten Modus in die Modi beschränkter Transpositionen ein. Seine Verwendung war selbst zu Nováks Zeit nichts Außergewöhnliches und Novák selbst nutzte ihn in anderen seiner Werke. Zu finden ist der oktatonische Modus etwa bei Rimsky-Korsakov, bei Janáček, beim jungen Stravinsky, usw.

12. Václav Štěpán, ,Symfonická tvorba“ [Symphonisches Werk], in Vitězslav Novák, Studie a vzpomínky [Studien und Erinnerungen], hrsg. Antonín Srba (Praha: Osvětový klub, 1932), 265. 
NotenBeispiel 1 Novák, Bouře, ab Ziffer 84
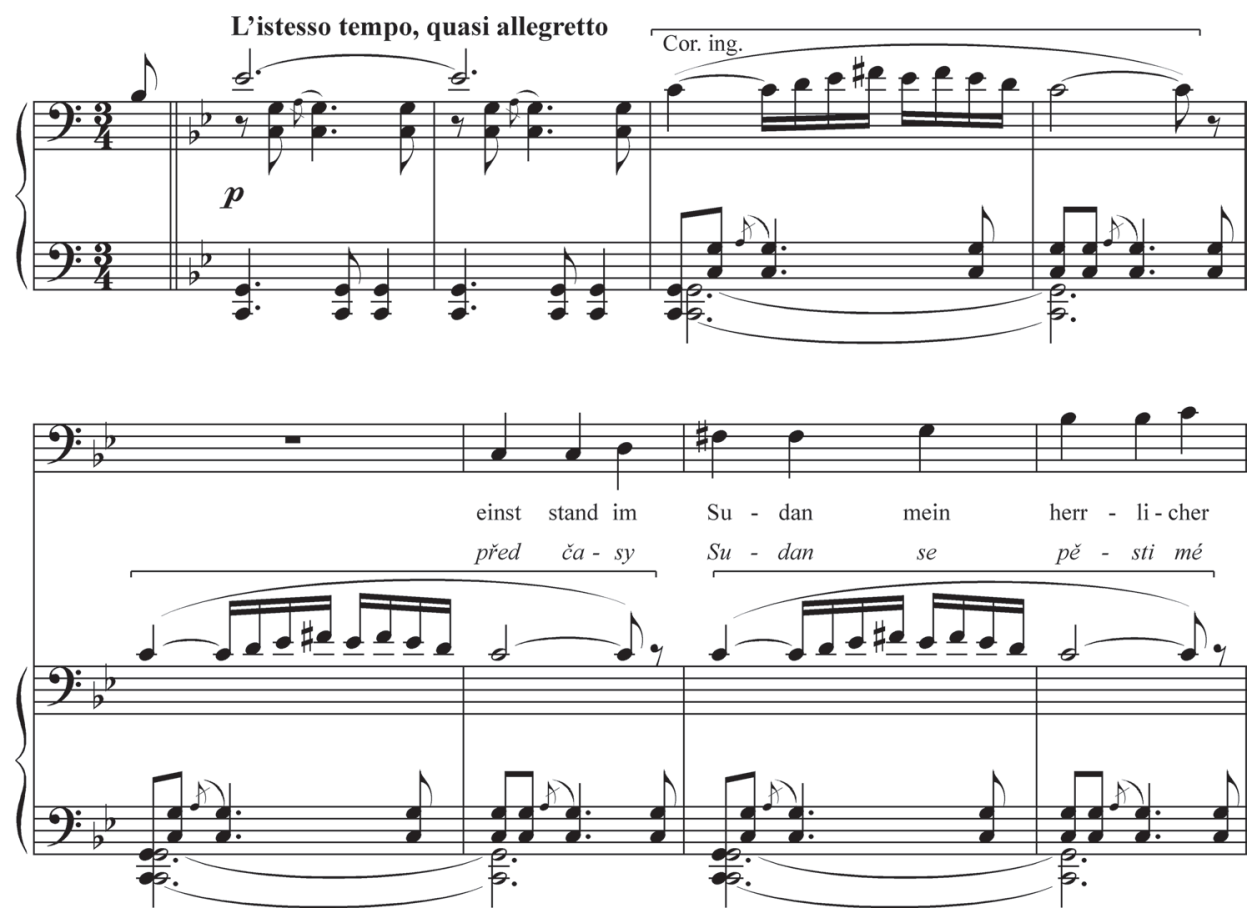

Die Ruhe des Wiegenlieds des Schwarzen, mit dem der Sklave seine Herrin zum Einschlafen bringt, ist jedoch trügerisch. So wie das Schiff im Sturm seinem unvermeidlichen Untergang entgegengeht, so steigert sich im Schwarzen Mann allmählich instinktive Leidenschaft, bis zu ihrem Ausbruch. Schließlich stürzt er sich auf seine wehrlose junge Herrin. So bestätigt sich das Klischee vom primitiven Schwarzen Mann, in dem verborgene Aggressionen glühen. In Grenzsituationen wird hier der ,,von nach Gewalt gierender Leidenschaft trunkene Sklave“"13 deshalb zu einem Gewalttäter und Sexualstraftäter an einer weißen Frau. ${ }^{14}$

MÄDCHEN: O, Sklave mein, ich fürchte dich!

Wahnsinn sprüht die Glut deiner Blicke!

SKLAVE: Schlaf', o Jungfrau, hör' mein Singen,

daß dich Träume hold umschlingen,

schlaf', o Herrin, schlaf':

13. Ebda., 248 .

14. Novák behauptet, dass er sich im Februar 1914 in Wien mit Franz Schrecker getroffen habe, der Interesse an der Aufführung des Sturmes gezeigt habe, allerdings ohne die ,shocking“ wirkende Szene in der Kajüte. Novák, O sobě a o jiných, 209. 
Auch dich, Schöne, Schwanenweiße, sinke Schlaf, der Ruh' verheiße dir,

o Herrin mein! Schlafe ein, schlaf' ein!

SKLAVE: Schläfst, mein Liebchen, schläfst zu lange.

MÄDCHEN: Laß mich, Satan droht im Blicke!

SKLAVE: Ich will küssen deine Wange.

MÄDCHEN: Laß mich! Laß los!

SKLAVE: Toll bin ich in Gier,

MÄDCHEN: Voller Tücke sind die Wogen.

SKLAVE: Liebchen,

MÄDCHEN: Ich ersticke!

SKLAVE: Liebchen, wach mit mir!

MÄDCHEN: O, Jesu Christ, o, Sklave mein! $!^{15}$

2.

Die Furcht vor kulturell fremden und auch fremd aussehenden Menschen bedingt sich wechselseitig mit dem Vorurteil, dass in anderen Kulturen die zivilisierte Selbstbeherrschung fehlt. Dies betrifft aber vor allem die Kategorie der unbekannten Fremden. Doch Jahrhunderte lang waren die Tschechen an erster Stelle von der Angst vor dem bekannten Fremden beherrscht. Das sind jene, die die Tschechen bereits gut kannten und seit jeher auf ihrem Territorium für Fremde gehalten hatten - also vor allem die Deutschen, also die westlichen Nachbaren.

Schon in den frühen Zeiten der tschechisch-deutschen Beziehungen kämpften die Tschechen mit Minderwertigkeitsgefühlen gegenüber ihren Nachbarn. Sie waren sich bewusst, dass deren entwickeltere Fertigkeiten zu einer Bedrohung wer-

15. Deutsche Übersetzung von Jan Löwenbach. 
den könnten, denn die Position des Nachahmers ist immer untergeordnet. Von der in Xenophobie gesteigerten Ambivalenz der tschechisch-deutschen Beziehungen zeugen bereits alte Chronik-Einträge in Versform, genannt auch die Empfehlung eines gewissen Dalimil, des Autors der ältesten tschechischen Chronik vom Beginn des 14. Jahrhunderts: ,Jeder bestellt sich Ratgeber zu seiner Regierung, doch einen Ausländer nur ein Dummkopf und Verräter."16 An anderer Stelle heißt es in der Chronik: „Meidet die Deutschen, wenn sie auch süß sprechen - wollt Ihr nicht einst am Strick hängen. ${ }^{\text {"17 }}$

Einen weiteren Meilenstein in den tschechisch-deutschen Beziehungen stellt die Zeit des tschechischen Hussitentums dar: Aus einer tschechischen Rebellion gegen die katholische Kirche entwickelte sich eine jahrzehntelange Kette aus Intrige, Verrat, Hass und beiderseitiger Aggression. Eine der langfristigen Folgen dieser Epoche war u. a. die Anregung der Reformation in Deutschland hundert Jahre später. Damit wiederum hängt die zahlreiche Zuwanderung von Deutschen nach Böhmen im 16. Jahrhundert zusammen. Dies erregte einigen Widerstand unter den Tschechen. Der tschechische Historiker František Palacký entwickelt im ersten Teil seiner Dějiny národu českého v Čechách i v Moravě (Geschichte der tschechischen Nation in Böhmen und Mähren) aus dem Jahr 1836 die These eines dauernden Konfliktes und der gegenseitigen Abgrenzung von Germanen und Slawen bzw. von Deutschen und Tschechen. Ängste vor den Tschechen und ihrem mutmaßlichen Streben nach der Gründung eines großslawischen Reiches, in dem das Germanentum vollkommen untergehen würde, wurden auf deutscher Seite bereits im Revolutionsjahr 1848 laut. Der Widerstand der Tschechen gegen die Deutschen erschien dort als eine Auflehnung wider die Natur. ${ }^{18}$

Es muss erwähnt werden, dass die Tschechen den Mut und die Ermunterung zu ihrer Emanzipationsbewegung paradoxerweise ebenfalls aus einer deutschen Quelle schöpften. Johann Gottfried Herder schildert im Kapitel über die slawischen Völker in seinem Hauptwerk Ideen zur Philosophie der Geschichte der Menschheit:

Sie [die Slawen] waren mildtätig, bis zur Verschwendung gastfrei, Liebhaber der ländlichen Freiheit, aber unterwürfig und gehorsam, des Raubens und Plünderns Feinde. ${ }^{19}[\ldots]$ Ist es ein Wunder, daß nach Jahrhunderten der Unterjochung und der tiefsten Erbitterung dieser Nation gegen ihre christlichen Herren und Räuber ihr weicher Charakter zur arglistigen, grausamen Knechtsträgheit herabgesunken wäre? ${ }^{20}$

16. Kronika tak řečeného Dalimila [Chronik des Pseudo-Dalimil] (Praha-Litomyšl: Paseka, 2005), 17.

17. Ebda., 192.

18. Alfred Payrleitner, Österreicher und Tschechen. Alter Streit und neue Hoffnung (Wien: Böhlau, 2/2003), 113. Der Autor zitiert hier die Allgemeine Zeitung vom 4. Juni 1848.

19. Johann Gottfried Herder, Ideen zur Philosophie der Geschichte der Menschheit (Berlin: Holzinger, 2013), 516.

20. Ebda., 517. 
Herders Worte waren aber auch Ursprung vieler späterer Vorurteile über die Tschechen: Sie seien fleißig, flexibel, sensibel, aber gleichzeitig unterwürfig, hinterhältig und frech. In den von beiden Nationen bewohnten Städten isolierten sich deren Angehörige zunehmend von- und gegeneinander. Sie schufen sich eigene, voneinander getrennte öffentliche Vereinigungen, Institute, Wohn- und Geschäftsviertel. Ein sehr gutes Beispiel dafür ist Olomouc, deutsch Olmütz, wo bis 1918 die deutschsprachige Mehrheit politisch und ökonomisch dominant war. Das Olmützer Stadttheater konnte in den 1860er Jahren noch regelmäßig von tschechischen Theatergesellschaften angemietet werden, doch in den 1880er Jahren waren tschechische Vorstellungen im deutschen Theater bereits unzulässig. Sie wurden in provisorische Räumlichkeiten jenseits der Stadtmauern verwiesen. In anderen binationalen Städten wie in Prag überwog wiederum die tschechische Bevölkerung. Hier wurden dem deutschen Teil der Bevölkerung ähnliche Kränkungen zugefügt. In Olmütz hatten Tschechen und Deutsche noch in der Zeit zwischen den Weltkriegen jeweils eine exklusive Promenade. Auch leisteten sich Tschechen und Deutsche jeweils einen eigenen städtischen Weihnachtsbaum auf dem Hauptplatz.

1918 wurde auf den Trümmern der Monarchie die Tschechoslowakische Republik gegründet. Die drei Millionen zählende deutsche Bevölkerung in den nördlichen oder nordwestlichen und südlichen Randregionen Böhmens und Mährens wollte aber nicht in diesem Staat leben. Die Abtrennung überwiegend deutschsprachiger Gebiete von der Tschechoslowakei wurde erst 1938 durchgesetzt. Den Weg dazu ebnete das von Hitler betriebene Münchner Abkommen der vier ausländischen Mächte Italien, Großbritannien, Frankreich und Deutschland. Die Tschechoslowakei war von den Verhandlungen über ihre Zukunft ausgeschlossen. Die Multikulturalität der jungen Tschechoslowakischen Republik wurde als eine ihrer Schwächen angesehen. Die spätere ethnische Homogenisierung dieses Landes war eine Folge der Vertreibung der Sudetendeutschen und der kommunistischen Isolation vom Westen. In Tschechien hält man diese ethnische und kulturelle Homogenität bis heute für einen Vorteil, den es zu schützen gelte. So jedenfalls erklärt der bulgarische Politologe Ivan Krastev die heutige, im Vergleich zu anderen westlichen Staaten zugespitzt ablehnende Haltung der Tschechen gegenüber der Migration aus Afrika und Asien. ${ }^{21}$ Diese Stationen und Einschnitte gehören zum Fundament des heutigen kollektiven tschechischen historischen Gedächtnisses. Sie stehen immer mit im Raum, wenn sich Tschechen mit den Deutschen als Fremden beschäftigen und ihnen begegnen.

21. Ivan Krastev, „Merklová jako pozdní Gorbačov“ [Merkel, der späte Gorbatschow], Lidové noviny (7. Juli 2018), 11-12. Ein weiteres Merkmal der Beziehung des Westens (im Gegensatz zum Osten) zu nationalen Minderheiten besteht laut Krastev in seiner kolonialen Vergangenheit. Während die Länder des Westens in der Zeit der Dekolonialisierung zu multikulturellen Gesellschaften geworden seien, hätten die östlichen Länder ihre kulturelle Homogenität gefestigt. 
Das Operndebüt Bedřich Smetanas Braniboři v Čechách (Die Brandenburger in Böhmen) ist eine Kondensation der tschechischen Ängste vor den Deutschen. Es geht um die Angst vor der deutschen Expansionskraft und der gewaltsamen Plünderung Böhmens, nicht zuletzt aber vor der Verdrängung des tschechischen Volkes und seiner Sprache. Der Stoff dieser Oper aus dem Jahr 1863 ist eng mit der ideologischen Orientierung des Komponisten verknüpft. ${ }^{22}$ Smetana war nämlich ein glühender Anhänger der so genannten Jungtschechen, die sich 1863 von der Nationalpartei, den so genannten Alttschechen, abgespalten hatten. Im Unterschied zu den Alttschechen, die mit František Palacký an der Spitze die Idee des Austroslawismus vertraten, also der Entwicklung der slawischen Völker im Rahmen der Donaumonarchie, strebten die Jungtschechen die radikale Abtrennung der Böhmischen Länder aus dem monarchistischen Verbund und ihre Befreiung von der Vorherrschaft des Kaisers und des deutschen Einflusses im habsburgischen Österreich. Der Librettist Karel Sabina situiert die Opernhandlung in die Zeit des Heranwachsens Václavs II., als in den Ländern der böhmischen Krone sein Vormund, der brandenburgische Markgraf Ota regierte. Václav II. bestieg den Thron erst später, im Alter von sieben Jahren. ${ }^{23}$

Nach einem kleinen Vorspiel befindet man sich auf dem Gut Volfram Olbramovičs. Hier erzählt Ritter Oldřich Rokycanský vom Wüten der Brandenburger in Böhmen:

OLDŘICH: Ich aber sage: nicht weiter sind zu dulden

die fremden Heere.

Zur Waffe greife man schon und vertreibe

die Brandenburger aus dem Vaterland,

denn sie plündern das Land,

verhöhnen unsere Sprache,

unter ihrem Schwert ächzt das Volk! $!^{24}$

Die Brandenburger haben Prag überfallen, die Stadt geplündert und den kleinen Václav mit seiner Mutter entführt. Der Prager Bürger Tausendmark ist der verschmähte Verehrer Ludišes, der Tochter Volframs. Er wird in seiner Kränkung zum Verräter der Stadt und zum Helfer der Brandenburger. Er stiftet sie zur Entführung der drei Volframschen Töchter, einschließlich Ludišes, an. Doch gegen die Brandenburger erhebt sich ein Volksaufstand und die Brandenburger müssen sich zurückziehen. Tausendmark kauft die Schwestern aus der Gefangenschaft

22. Auf diese Tatsache weist neuerdings auch Kelly St. Pierre hin: Bedřich Smetana. Myth, Music, and Propaganda (Rochester: University of Rochester Press, 2017).

23. Václav II. (1271-1305), böhmischer und polnischer König aus der Dynastie der Premysliden, regierte in den Jahren 1278/83-1305.

24. Deutsche Übersetzung von Jens-Peter Cyprian. 
frei und will mit den Brandenburgern in die Fremde ziehen. Nach vielen Peripetien werden die Schwestern gerettet. Der Verräter Tausendmark muss sich vor Gericht verantworten; die Brandenburger werden schließlich aus dem Land vertrieben. Die eher pseudohistorische, aber ideologisch antideutsche Handlung machte es dieser Oper natürlich unmöglich, sich auf deutschen Bühnen durchzusetzen. In Deutschland wurden die Brandenburger in Böhmen erstmals 1994 in Bautzen aufgeführt, 14 Jahre nach ihrer britischen Premiere.

Ein in dieser Hinsicht glücklicheres Schicksal traf Dvořáks Jakobiner, ein weiteres Werk aus dem tschechischen Opern-Grundrepertoire. In ihr könnte man weitere Belege für das tief verwurzelte Misstrauen gegenüber dem Fremden finden, welches der tschechischen Erfahrung so entfernt ist. Der symbolische Ausdruck dieses Misstrauens ist von Smetanas Radikalität allerdings weit entfernt. Die alttschechische Gedankenwelt, zu deren vordersten Repräsentanten auch František Rieger gehörte, der Koautor des Librettos des Jakobiners, stand Dvořáks Naturell nämlich sehr viel näher als Smetana. Gerade das urtschechische Sujet und die sich ihm anschließende musikalische Verarbeitung mit typisch tschechisch klingenden Melodien kennzeichnen diese Oper; im Sinne der national gemäßigten Alttschechen kämpft Dvořák also mit einem Liedchen für die tschechischen Interessen. Dies führte später zur Stigmatisierung der Oper als „Volksoper“ im guten wie im schlechten Sinn. Es ist gut bekannt, dass Die verkaufte Braut in Nazi-Deutschland äußerst erfolgreich war. Aber auch der Jakobiner mit seiner Ideologie von Volkstum und Emotionalität fand dort Anklang. Die Kritik erwähnt nach der Dresdner Premiere der Jakobiners Dvořáks, dass „,darin ohne blasse ästhetisierende Problematik aus einem vollen Musikantenherzen musiziert wird“". 25 Andererseits galt Dvořák weiterhin als Repräsentant des einfachen tschechischen Musikantentums. Dazu trug auch die langfristige Bewertung Dvořáks durch einen Teil der deutschen Musikwissenschaft bei. In seinem musikalischen Genie sah man einen Beweis für Beschränkung durch Rassenzugehörigkeit: seine Musik, so schreibt Hans-Joachim Moser im Jahre 1944, sei „nur Wasser und nie Wein“. ${ }^{26}$

Obwohl sich diese Bewertungen heute in keiner Weise mehr ernst nehmen lassen, zeigt sich im Rahmen der Handlung der Oper doch eine gewisse Beschränkung auf eine abgeschlossene tschechische Umgebung: eine tschechische Kleinstadt zur Zeit der französischen Revolution ist der sowohl lokale, als auch geistige Horizont der Auseinandersetzung mit dem Fremden. Eines der wichtigsten Elemente der Opernhandlung wird das Motiv des tschechischen Musikantentums. Das tschechische Lied ist Symbol der nationalen Kraft und des Patriotentums der Kleinstadtbewohner. Es unterstützt auch die Verstoßenen Bohuš und Julie bei

25. Anonym, Leipziger Neueste Nachrichten (7. Februar 1943), 3, zitiert nach Klaus Döge, Antonín Dvořák. Leben. Werke. Dokumenten (Zürich-Mainz: Atlantis Musikbuch-Verlag, 2/1997), 427-428.

26. Hans Joachim Moser, „Anton Dvorak“, Strassburger Neueste Nachrichten (2. Mai 1944). Zitiert nach Döge, Antonín Dvořák, 428. 
ihrer langen Wanderung durch die Fremde. In ihrem berühmten Duett im zweiten Akt heißt es:

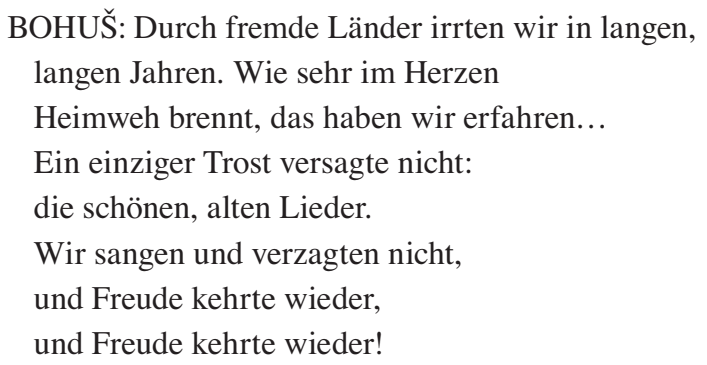

Für die Stadtbewohner sind Bohuš und Julie aber nur verdächtige Fremdlinge, die aus dem entfernten Ausland kommen; sie stehen außerdem im Verdacht, Jakobiner zu sein, Verkünder des gefährlichen Freidenkertums und Vertreter des Chaos. So will der Schlossherr der Stadt die beiden nicht ziehen lassen, bevor sie sich nicht legitimiert haben:

SCHLOSS: Mir scheint da stimmt etwas nicht!

He, auf ein Wörtchen!

Sagt, was sucht Ihr in der Stadt hier?

Euren Ausweis! Woher kommt Ihr?

Zeigt mir Euren Paß! ${ }^{27}$

In der allgemeinen Katharsis, während der großen Versöhnung des Grafen, des Vaters von Bohuš, mit seinem verlorenen und früher verfluchten Sohn, stellt sich zur allgemeinen Zufriedenheit Folgendes heraus: die Haupthelden sind keine Jakobiner und mussten im Gegenteil vor der jakobinischen Böswilligkeit zurück in die Heimat fliehen. Alles kehrt also zur gehörigen Ordnung zurück: Aus den Fremdlingen werden die Unsrigen und die althergebrachten Strukturen der Stadt werden nicht weiter von außen gestört. Das Fremde wird letztendlich in Wohlgefallen aufgelöst, es gibt in letzter Konsequenz nur ,uns“.

In Dvořáks Rusalka zum Libretto von Jaroslav Kvapil sind gleich zwei Figuren als Fremdelemente anzusehen: die fremde Fürstin (höchstwahrscheinlich eine Deutsche) und Rusalka. Beide stellen jeweils zwei gegensätzliche Welten dar: Die Fürstin auf der einen Seite steht für die menschliche Welt mit allen ihren charakterlichen Schwächen und Fehlern, Rusalka andererseits repräsentiert die ideale und reine Welt der Märchen. Sie ist den Ortsansässigen als verdächtiges Wesen nicht willkommen. Denn der Prinz hat sie sich nur von irgendwoher aus dem Wald

27. Übersetzung ins Deutsche von Kurt Honolka. 
mitgebracht. Ihre Andersartigkeit ruft Ablehnung und gleichzeitig Schrecken hervor. Die Fürstin ist die ausdrücklich negative Gestalt der gesamten Handlung. Den Prinzen liebt sie nicht, will ihn aber für sich gewinnen und von Rusalka weglocken:

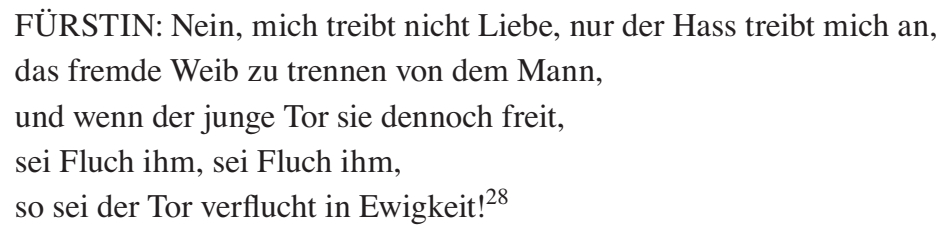

Dazu nutzt sie die Wankelmütigkeit des Prinzen aus. Nachdem sie ihr Ziel erreicht hat, verlässt sie ihn aber wieder. Die Folgen ihres Handelns sind tragisch. Antipathien gegen die Fürstin werden durch die Betonung ihrer Herkunft noch potenziert: sie ist eine „fremde Fürstin“, fremd und böse werden hier zu Synonymen.

Das Fremde in der tschechischen Musik bedeutet Faszination und Abscheu. Fremd sind einerseits Unterdrücker und Eroberer, fremd sind aber auch die Heilsbringer und Glücksversprechen exotischer Welten. Die Erfahrung der Ambivalenz des Fremden ist sicher nicht auf Tschechien beschränkt. Weltweit ist das Fremde Projektionsfläche von Wünschen und Ängsten, Gegenstand von Fantasie und Verdrängung. Ähnliche Motive könnten wir auch in einer Reihe weiterer Kunstwerke unterschiedlicher Provenienz finden. Im Falle der kleinen Nationen wird das Fremde allerdings in der Konstellation konkreter historischer Situationen behandelt und aus der Perspektive ihrer überlieferten Interpretationen. Die virulenten Traumata einer Nation steigen in einer angespannten politischen Atmosphäre an die Oberfläche und beginnen dann aktuell zu wirken. Es scheint jedoch, dass das Misstrauen gegenüber dem Fremden zu einem bedeutenden Teil auf der nationalen Kultur und Identität der Tschechen beruht, egal, aus welcher Himmelsrichtung das Fremde kommt. 